\title{
"Cyst-like" structures within the ciliary shafts in children with bronchiectasis
}

\author{
M. Pifferi*, A.M. Cangiotti ${ }^{\#}$, D. Caramella ${ }^{\Uparrow}$, A. Pietrobelli ${ }^{+}$, V. Ragazzo*, E. De Marco*, P. Macchia*, \\ S. Cinti ${ }^{\#}$, A.L. Boner ${ }^{+}$
}

"Cyst-like" structures within the ciliary shafts in children with bronchiectasis. M. Pifferi, A.M. Cangiotti, D. Caramella, A. Pietrobelli, V. Ragazzo, E. De Marco, P. Macchia, S. Cinti, A.L. Boner. (C) ERS Journals Ltd 2004.

ABSTRACT: "Cyst-like" structures within the ciliary shafts were considered in four adults as a primary defect involved in the development of bronchiectasis. In this study, the presence and the primary or secondary nature of this abnormality were assessed in children with bronchiectasis.

High resolution computed tomography (HRCT) and nasal biopsies for motion analysis and transmission electron microscopy (TEM) evaluation of cilia were obtained in 45 children with recurrent lower airway infections and abnormal chest radiography.

HRCT disclosed bronchiectasis in 35 out of $45(77.8 \%)$ children and cyst-like structures were demonstrated with TEM in 29 out of $45(64.4 \%)$ patients. Cyst-like structures were constantly associated with other ultrastructural abnormalities commonly observed in chronic inflammation, and were found both in subjects with primary and with secondary ciliary dyskinesia. When considering only patients with bronchiectasis, a significant correlation between prevalence of cyst-like structures and the severity of bronchiectasis was demonstrated. Follow-up (2-22 months) of seven patients demonstrated that in the five children with secondary dyskinesia, the ultrastructural defect completely disappeared and there was a small reduction in the abnormality in the two patients with primary dyskinesia.

In contrast to one previous report, the reversibility of the defect suggests its secondary origin, which is most likely related to chronic airway inflammation. Eur Respir J 2004; 23: 857-860.
Depts of *Pediatrics and ${ }^{\top}$ Radiology, University of Pisa, Pisa, ${ }^{\#}$ Institute of Normal Human Morphology, Electron Microscopy Unit, University of Ancona, Umberto $\mathrm{I}^{\circ}$ Hospital, Ancona, and, ${ }^{+}$Dept of Pediatrics, University of Verona, Verona, Italy.

\section{Correspondence: M. Pifferi}

Dept of Pediatrics

University of Pisa

Via Roma 67

56100 Pisa

Italy

Fax: 3950888622

E-mail: m.pifferi@med.unipi.it

Keywords: Bronchiectasis children

cyst-like structures

high resolution computed tomography transmission electron microscopy

Received: July 242003

Accepted after revision: January 212004
Impairment of mucociliary transport in patients with ciliary ultrastructural abnormalities, such as in primary ciliary dyskinesia (PCD), usually results in the development of early-onset bronchiectasis due to recurrent respiratory tract infections [1-5]. Occasionally, patients with PCD show a normal ciliary ultrastrucural pattern with abnormal ciliary function, which may also lead to airway remodelling [6, 7]. Recently, a new ciliary ultrastructural defect was identified in four adults with severe idiopathic bronchiectasis [8]. The abnormality consisted of "cyst-like" structures located at the base of the cilia with the association of a small defect in function [8].

The absence of other ultrastructural defects of the cilia, and of other possible causes for bronchiectasis, allowed the authors to consider the newly described abnormality as a primary defect possibly involved in the development of bronchiectasis [8]. However, adult patients may not be ideal to evaluate early-onset bronchiectasis and its association with an underlying genetic condition.

The aims of this study were to assess the correlation of cystlike ciliary defects with the presence and severity of bronchiectasis, and to ascertain the primary or secondary nature of this ultrastructural defect in a prospectively recruited paediatric cohort.

\section{Materials and methods}

A total of 45 consecutive patients (22 males, 23 females) aged $0.6-18.0$ yrs (mean \pm SD 7.6 \pm 4.5 ), suffering from recurrent lower airways infections and with chest radiographs with features of bronchiectasis, were enrolled between June 2000 and February 2003.

Seven patients had primary cilia dyskinesia (three with Kartagener's Syndrome), one had cystic fibrosis and 37 had recurrent bronchopneumonia (four of them were also asthmatic and nine also had recurrent otitis media and sinusitis).

\section{High resolution computed tomography}

In all patients a high resolution computed tomography (HRCT) was performed using a third-generation scanner (Sytech 3000; General Electric Medical Systems, Milwaukee, MI, USA). Slices (1-mm thick) were obtained with 10-mm spacing, in the supine position, at full inspiration [9]. No intravenous contrast was injected and anaesthesia was needed in three patients. All images were reviewed by the same radiologist who was blinded to the clinical data. Bronchiectasis was scored from: 1) mild bronchial dilatation found in limited parenchymal areas; to 3 ) severe and diffuse bronchiectatic changes (fig. $1 \mathrm{a}, \mathrm{b}, \mathrm{c}$ ), according to the extent of disease. Subjects with HRCT score 0 (no bronchiectasis) were used as controls.

\section{Cilia evaluation}

In all patients, both ciliary motion analysis and transmission electron microscopy (TEM) evaluation of cilia were 
performed. Biopsies were obtained on nasal ciliated epithelium from two or more sites of the inferior turbinate. No patient reported respiratory infections or exacerbations of the chronic disease in the previous month, and medication was not taken in the $48 \mathrm{~h}$ prior to the study. Samples obtained using a cytology brush (Microvasive, Milford, MA, USA), were suspended in $2 \mathrm{~mL}$ Medium 199 fluid cell culture and in $2 \mathrm{~mL}$ normal saline for immediate light-microscopic and TEM evaluation, respectively [10]. Samples for ciliary motion analysis were kept at $37^{\circ} \mathrm{C}$ and transferred to a variablethickness culture chamber [11]. Ciliary morphology, motion pattern and beat frequency were evaluated according to standardised methodology [2, 3, 7, 12].
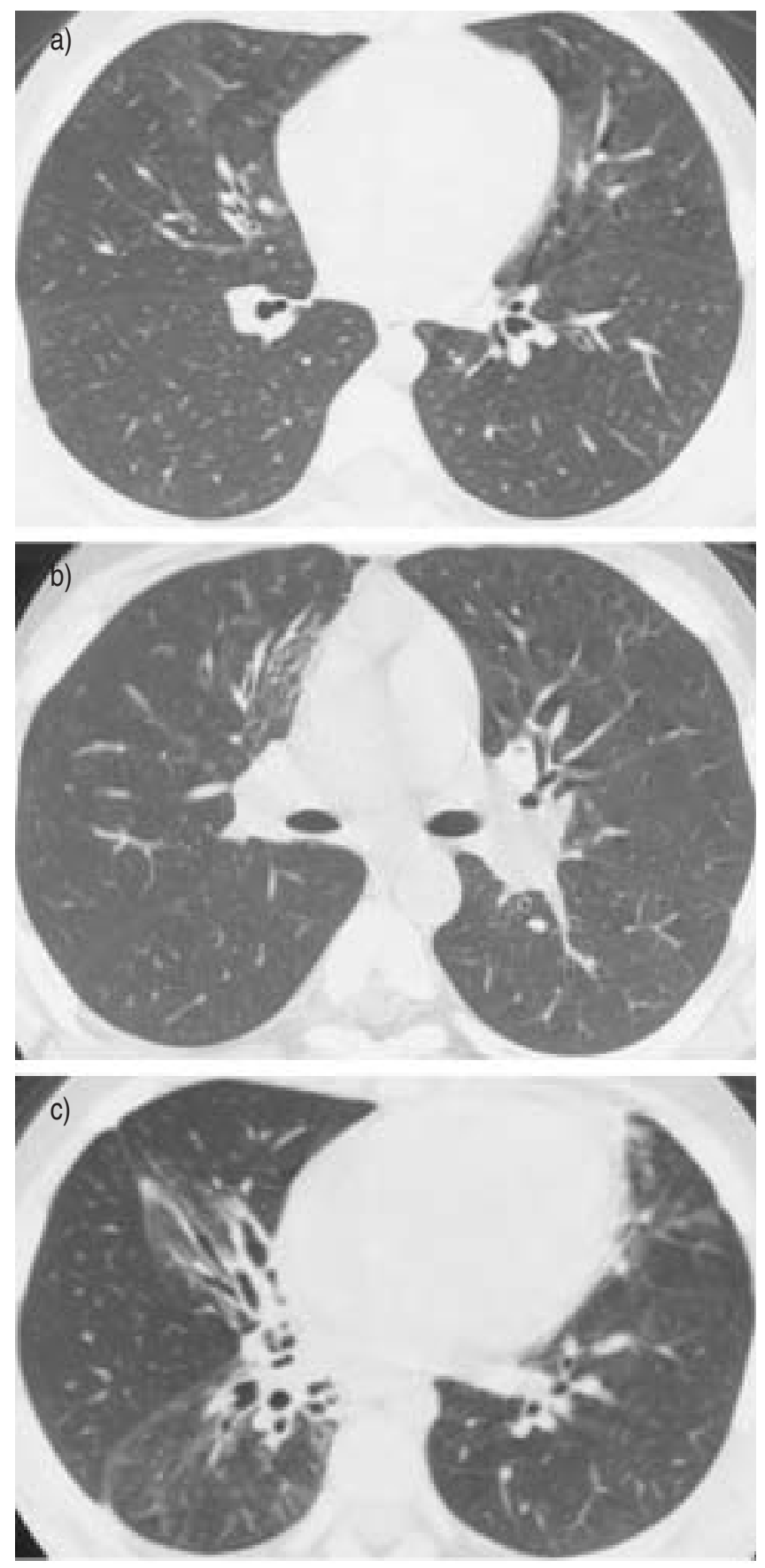

Fig. 1.-Score of bronchiectasis: a) mild bronchial dilatation found in limited parenchymal areas (score 1); b) intermediate bronchiectasis (score 2); c) severe and diffuse bronchiectatic changes (score 3).
Samples for ultrastructural studies were also prepared according to standardised methodology [13]. Cilia were studied at a final magnification of $\times 103,000-207,000$. For each specimen, an average of 14 ciliated cells and 120 random sections of cilia were examined to assess the presence of cystlike structures within the ciliary shafts and any other structural defects. Ciliary motion analysis and ultrastructural evaluation were independently performed by two different experts.

In seven patients, it was possible to re-evaluate ciliary beat frequency and ciliary ultrastructure after a follow-up period of 2-22 months of aggressive treatment according to a standardised protocol [3].

\section{Other analyses}

To evaluate possible causes of bronchiectasis, additional tests were performed [5]: the sweat test; $\alpha_{1}$-antitrypsin; quantitative immunoglobulin (Ig); IgG subclasses; total IgE; leukocyte counts with differential cell counts; neutrophil chemotaxis; phagocytosis; neutrophil killing; T-lymphocyte subclasses, C3, C4 and CH50. Finally, congenital malformations (i.e. Williams-Campbell syndrome, cystic adenomatoid or hamartomatous malformations, etc.) were studied by analysis of the HRCT images acquired for the evaluation of the bronchiectasis.

Informed parental consent was obtained before enrolling the children and the study protocol was approved by the local hospital ethical committee.

\section{Statistical analyses}

Baseline variables are described as group mean \pm SD. Sensitivity, specificity, positive predictive value and negative predictive value of cyst-like structures within the ciliary shafts, as indicators of HRCT-proven bronchiectasis, were calculated. The statistical significance of correlations between the results obtained with TEM and HRCT were examined using Spearman's Rank Signed test. A p-value $<0.05$ was considered statistically significant.

\section{Results}

Ciliary motion analysis (abnormal motion patterns, including immotile cilia and/or ciliary beat frequency $<6 \mathrm{~Hz}$ ) and TEM evaluation of cilia (alterations of the central pair and dynein arms deficiencies, associated with a small proportion of swollen cilia and compound cilia) confirmed the diagnosis of PCD in seven children (15.5\%). According to the clinical phenotype, three of them had Kartagener's syndrome [3, 6]. In the remaining 38 patients $(84.5 \%)$, ciliary motion analysis demonstrated abnormal patterns in a small proportion of cilia, prevalence of thick cilia and low ciliary beat frequency, although $>6 \mathrm{~Hz}(7.9 \pm 1.6 \mathrm{~Hz})$, compatible with secondary ciliary dyskinesia (SCD) $[2-4,7]$. In these subjects, TEM evaluation showed nonspecific abnormalities compatible with chronic inflammation (prevalence of swollen cilia and compound cilia).

Cyst-like structures within the ciliary shafts were demonstrated in 29 out of 45 patients $(64.4 \%)$ and ultrastructural analysis showed that $0.7-37.5 \%$ of cilia displayed such a defect with variable size (fig. 2). The defect was present both in patients with primary and secondary ciliary dyskinesia, and also in one patient with no documented bronchiectasis.

In 10 subjects, no bronchiectasis (HRCT score of 0 ) could 
be demonstrated. All the children had recurrent bronchopneumonia, three also had asthma and one also had sinusitis. The clinical phenotypes of these patients were not different from the $35(77.8 \%)$ patients with bronchiectasis, obviously excluding the three patients with situs inversus viscerum. The bronchiectasis scores for HRCT in these 35 patients were 1,2 and 3 , in 14,13 , and eight patients, respectively.

Individual percentages of cilia with a cyst-like lesion in relation to bronchiectasis HRCT score are reported in figure 3. This figure depicts the subjects with PCD (seven patients) and the subjects with SCD (28 patients).

The presence of cyst-like structures, as an indicator of HRCT-proven bronchiectasis in all patients together (PCD and SCD), had a sensitivity of $80 \%$ and a specificity of $90 \%$. The positive predictive value was $96.6 \%$ and negative predictive value $56.2 \%$. In subjects with SCD, sensitivity was $78.6 \%$ and specificity $90 \%$. Positive predictive value was $95.6 \%$ and negative predictive value $60 \%$. Spearman Rank Signed correlation test demonstrated a significant correlation between the per cent of cyst-like structures within the ciliary shafts and the HRCT score $(\mathrm{p}=0.039)$. No immunological defects, including functional assessment (response to antipolio vaccine), nor $\alpha_{1}$-antitrypsin deficiency were found in the

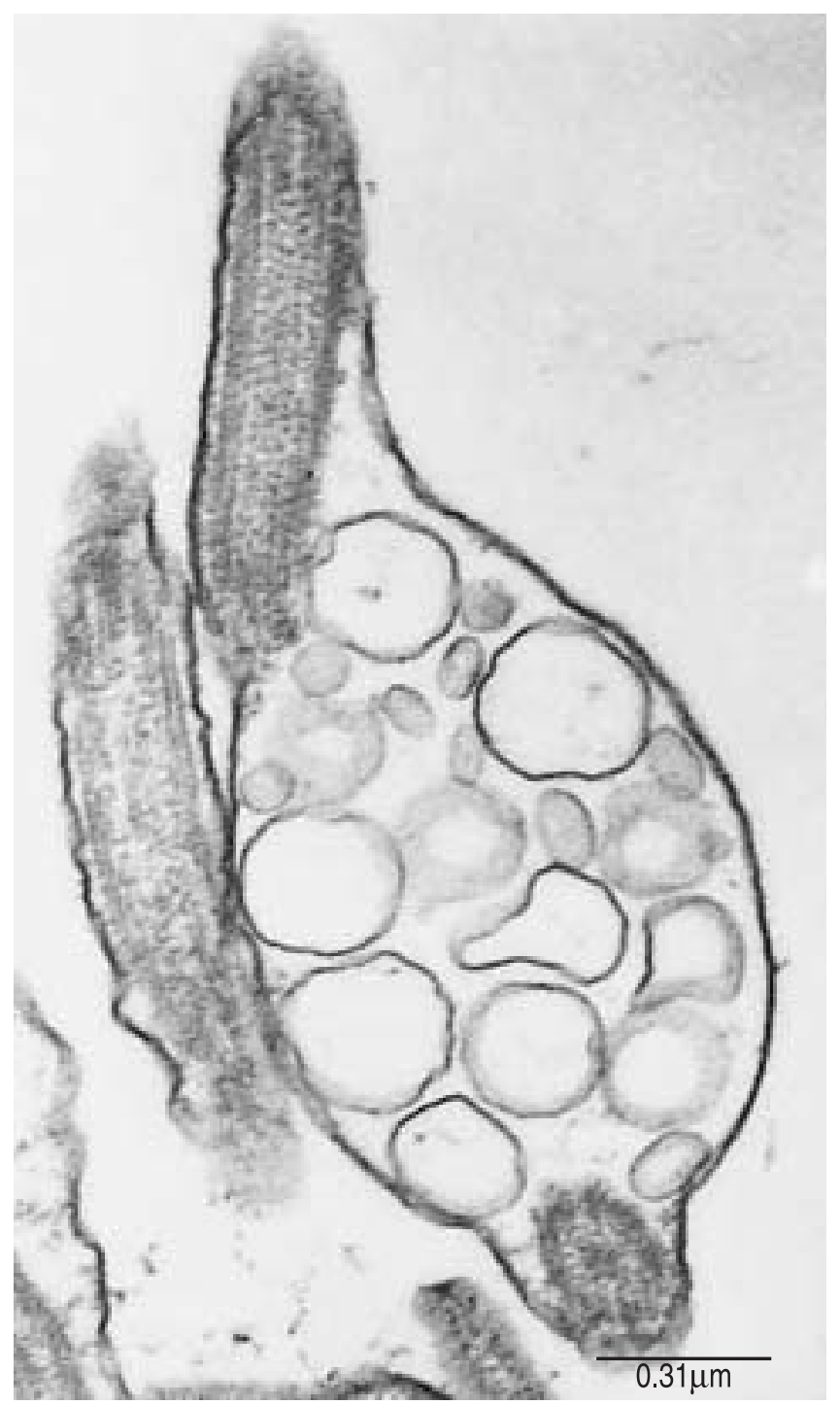

Fig. 2. - Cyst-like structures within the ciliary shafts.

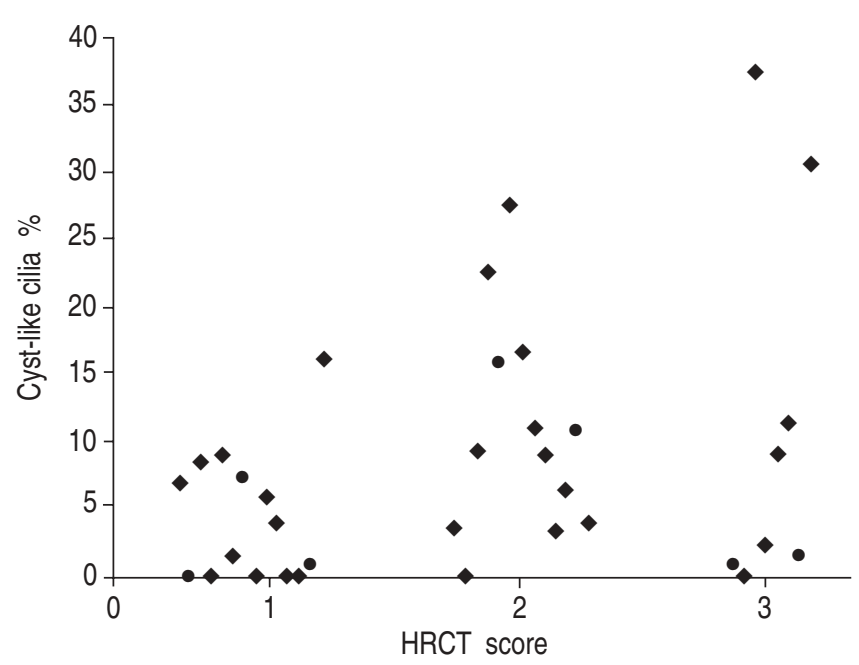

Fig. 3.-Percentage of cilia with cyst-like lesions in relation to high resolution computed tomography (HRCT) score for bronchiectasis (35 subjects). O: primary ciliary dyskinesia; $\bullet$ : secondary ciliary dyskinesia.

present study population. Furthermore, no congenital malformation was observed at HRCT.

Seven patients were re-evaluated 2-22 months after the initial examination. During this period they received antibiotic treatment and chest physiotherapy. In five cases, the cyst-like structures disappeared. These five patients had SCD and the HRCT scores were: 1 in two patients, 2 in one child and 3 in two subjects. In the remaining two patients with PCD and a HRCT score of 2, the defect persisted although with a reduced intensity (from 15.8 to $6.8 \%$ and from 10.8 to $7.3 \%$ of the cilia). In these two patients the follow-up was 3 months for one patient and 2 months for the other.

\section{Discussion}

Cyst-like structures within the ciliary shafts were described for the first time in four adult patients with otherwise normal microtubular ultrastructure by TSANG et al. [8]. The authors attributed a pathogenetic role to this defect in the development of bronchiectasis and hypothesised that these cyst-like structures were a primary defect on the basis of their persistence in the 3-12 months follow-up [8].

In the children in the current study, cyst-like structures were demonstrated both in subjects with idiopathic bronchiectasis and in patients with bronchiectasis related to PCD. Furthermore, after antibiotic therapy given during the followup, the defect completely disappeared in the five children with SCD and was reduced in the two patients with PCD. Although the follow-up was only performed in seven patients, the disappearance of the defect suggest that these cyst-like structures are an expression of the chronic airway inflammation that often occurs with other ultrastructural abnormalities $[7,14]$. Data on sputum microbiology was not obtained in the current study, so no specific bacterial infection can be related to the detected abnormalities. It is also unlikely that the cystlike appearance is due to an artefact of tissue specimen processing, since if this was the case it should have occurred both at the beginning and at the follow-up evaluation. Furthermore, the possibility of an error in the evaluation of biopsies of cilia was reduced by the use of two independent observers looking at 120 random sections of cilia. In addition, the functional abnormalities found in all patients with different conditions other than PCD are most probably 
related to chronic inflammation and are classified as SCD [7, 15-17]. Cyst-like structures may effectively be a consequence of deciliation by harmful agents, as previously reported for different abnormalities in humans and experimental animals $[18,19]$. Therefore, the meaning of these alterations might be nonspecific, comparable to that of compound cilia, of membrane-deficient cilia and of giant cilia [17, 20]. In fact, even in the absence of PCD, some viral infections can cause a transient but severe impairment of mucociliary clearance for up to 4 months [21]. Moreover, some bacterial infections (Pseudomonas sp., Haemophylus, Pneumococci, Chlamydiae and Mycoplasma) are ciliotoxic and cause reversible ciliostasis, which may impair mucociliary clearance for months [22-24].

To further support the authors' hypothesis, a reduction in the percentage of defected cilia was also observed in the two patients with PCD. In those patients where it was not completely reversed, a follow-up of $2-3$ months is probably too short a period to show a significant reduction in the structural defect in the presence of a disease usually associated with severe airway inflammation. Equally, disease duration and severity in patients described by TsANG et al. [8] may explain the persistence of cilia abnormalities.

Although it may be suggested that these basally situated cyst-like structures could affect, primarily, the beating of the cilia itself and secondarily that of the neighbouring cilia [8], it is also possible that the functional impairment is a global consequence of inflammation. This was previously observed for swollen and compound cilia [7, 14, 15]. This also seems to be the case for cyst-like lesions in consideration of the complete or partial reversibility of the ultrastructural defect in SCD and PCD, respectively. Furthermore, the high specificity and high positive predictive value of cyst-like cilia structures as an indicator of the presence of bronchiectasis in children with both PCD and SCD represent a strong argument in favour of its inflammatory origin. However, the significant correlation between TEM cilia dysfunction and HRCT score suggests that the percentage of the affected cilia, even though not pathogenetic, might have a prognostic impact on the clinical conditions. As a consequence, their identification requires an appropriate aggressive management plan to prevent and possibly reverse airways damage [3, 7].

In conclusion, in children, ultrastructural changes of the cilia are secondary to chronic infection and can be resolved after appropriate long-term treatment.

\section{References}

1. Afzelius BA. A human syndrome caused by immotile cilia. Science 1976; 193: 317-319.

2. Schidlow DV. Primary ciliary dyskinesia (the immotile cilia syndrome). Ann Allergy 1994; 73: 457-469.

3. Bush A, Cole P, Hariri M, et al. Primary ciliary dyskinesia: diagnosis and standards of care. Eur Respir J 1998; 12: 982 988.

4. Afzelius BA. Immotile cilia syndrome: past, present, and prospects for the future. Thorax 1998; 53: 894-897.

5. Pasteur MC, Helliwell SM, Houghton SJ, et al. An investigation into causative factors in patients with bronchiectasis. Am J Respir Crit Care Med 2000; 162: 1277-1284.
6. Greenstone M, Rutman A, Pavia D, Lawrence D, Cole PJ Normal axonemal structure and function in Kartagener's syndrome: an explicable paradox. Thorax 1985; 40: 956957.

7. Pifferi M, Cangiotti AM, Ragazzo V, Baldini G, Cinti S, Boner AL. Primary ciliary dyskinesia: diagnosis in children with inconclusive ultrastructural evaluation. Pediatr Allergy Immunol 2001; 12: 274-282.

8. Tsang KWT, Tipoe G, Sun J, et al. Severe bronchiectasis in patients with "cystlike" structures within the ciliary shafts. Am J Respir Crit Care Med 2000; 161: 1300-1305.

9. McGuinness G, Naidich DP, Leitman BS, McCauley DI Bronchiectasis: CT evaluation. Am J Roentgenol 1993; 160: 253-259.

10. Rutland J, Cole PJ. Non-invasive sampling of nasal cilia for measurement of beat frequency and study of ultrastructure. Lancet 1980; 2: 564-565.

11. Braga PC. A variable-thickness, multipurpose culture chamber for high-magnification observation. $J$ Microscopy 1990; 159: 285-288.

12. Rossman CM, Forrest JB, Lee RMKW, Newhouse MT. The dyskinetic cilia syndrome. Ciliary motility in immotile cilia syndrome. Chest 1980; 78: 580-582.

13. Carlén B, Stenram U. Ultrastructural diagnosis in the immotile cilia syndrome. Ultrastruct Pathol 1987; 11: 653658.

14. Rossman CM, Lee RMKW, Forrest JB, Newhouse MT Nasal ciliary ultrastructure and function in patient with primary ciliary dyskinesia compared with that in normal subjects and in subjects with various respiratory diseases. $\mathrm{Am}$ Rev Respir Dis 1984; 129: 161-167.

15. Wilson R. Secondary ciliary dysfunction. Clin Sci 1988; 75: 113-120.

16. Buchdahl RM, Reiser J, Ingram D, Rutman A, Cole PJ, Warner JO. Ciliary abnormalities in respiratory disease. Arch Dis Child 1988; 63: 238-243.

17. Lurie M, Rennert G, Goldenberg S, Rivlin J, Greenberg E, Katz I. Ciliary ultrastructure in primary ciliary dyskinesia and other chronic respiratory conditions: the relevance of microtubular abnormalities. Ultrastruct Pathol 1992; 16: 547-553.

18. Fonzi L, Lungarella G, De Santi MM. Ultrastructural observations on morphogenesis of atypical cilia. Anat Anz Jena 1982; 151: 151-159.

19. Hagiwara H, Ohwada N, Aoki T, Takata K. Ciliogenesis and ciliary abnormalities. Med Electron Microsc 2000; 33: $109-114$.

20. Afzelius BA. "Immotile cilia" syndrome and ciliary abnormalities induced by infection and iniury. Am Rev Respir Dis 1981; 124: 107-109.

21. Gerrard CS, Levandowski RA, Gerrity TR, Yeates DB, Klein $\mathrm{E}$. The acute effects of acute respiratory virus infection upon tracheal mucous transport. Arch Environ Health 1985; 40: $322-325$.

22. Wilson R, Cole PJ. The effect of bacterial products on ciliary function. Am Rev Respir Dis 1988; 138: S49-S53.

23. Shemer-Avni Y, Lieberman D. Chlamydia pneumoniaeinduced ciliostasis in ciliated bronchial epithelial cells. J Infect Dis 1995; 171: 1274-1278.

24. Fujihara K, Yamanaka N, Bernstein JM, Ogra PL, Hard R. Morphologic and motility changes of nasal cilia in primary culture caused by Haemophilus influenzae. Ann Otol Rhinol Laryngol 1996; 105: 452-457. 